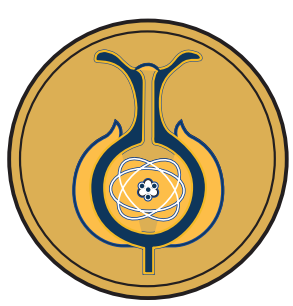

Determinación de intervalos básicos de salud en tortugas resbaladoras (Trachemys scripta emolli) del Refugio de Vida Silvestre Caño Negro, Costa Rica

Determining basic health intervals in tortugas resbaladoras (Trachemys scripta emolli) of the Caño Negro Wildlife Refuge, Costa Rica

Determinação de intervalos básicos de saúde em tortugas resbaladoras (Trachemys scripta emolli) do Refúgio de Vida Selvagem Caño Negro, Costa Rica

Laura Fournier-Gutiérrez

mfglaura@gmail.com

Instituto Costarricense de Electricidad, San José, Costa Rica.

http://Orcid.org/0000-0002-1599-1666

\section{Rosibel Barrantes-Barrantes}

rosibelb@gmail.com

Investigadora privada,

San Carlos, Costa Rica.

http://Orcid.org/0000-0003-1070-1698
Marco V. Herrero

herrero1958@hotmail.com

Posgrado Regional en Ciencias Veterinarias, Escuela de

Medicina Veterinaria,

Universidad Nacional,

Heredia, Costa Rica.

http://Orcid.org/0000-0001-6964-4048

Kinndle Blanco-Peña

kblanco@una.ac.cr

Posgrado Regional en Ciencias Veterinarias, Escuela de

Medicina Veterinaria,

Instituto Regional de Estudios en Sustancias Tóxicas,

Universidad Nacional,

Heredia, Costa Rica.

http://Orcid.org/0000-0002-0883-1832

Received: 9/Aug/2019 • Accepted: 18/Set/2019・Published: 31/Jul/2020

\title{
Resumen
}

El objetivo de este trabajo es determinar los parámetros sanguíneos y la presencia de parásitos en una población salvaje de tortugas deslizantes (Trachemys scripta emolli), durante la estación seca de 2013, en el Refugio de Vida Silvestre Caño Negro, Costa Rica. Utilizando redes, los individuos fueron capturados de diferentes huecos. Se tomó muestras de sangre para determinar los parámetros químicos de esta y el hematocrito, así como los valores morfológicos y las condiciones corporales. Además, se recolectaron muestras de heces y ectoparásitos. El IC del hematocrito $95.0 \%$ fue [14 7091, 18 7356] y no se encontraron diferencias significativas entre los sexos. Los IC del $95 \%$ para los parámetros de la química sanguínea fueron: AST(U/L) [87 6214, 148 929], CK (U/L)[1439.2, 2487.08], AU (umol/L) [68.22, 90.43], GLU (mmol/L)[4.26, $5.47]$, PHOS [0.46, 0.61], Ca (mmol/L) [1.99, 2.15], PT ( $\mathrm{g} / \mathrm{L})[22.16,24.44], \mathrm{K}(\mathrm{mmol} / \mathrm{L})[3.31,3.83$ ] y Na (mmol/L)[127.92,130.93]. También se obtuvo 21 muestras de heces, de las cuales 10 fueron positivas para 
parásitos (23.8 \% Strongyloides, $14.3 \%$ Ascaridia, $9.5 \%$ Coccidia y $9.5 \%$ Pentastomida). Una sanguijuela de la familia Rhynchobdellidae fue el único ectoparásito encontrado (Placobdella sp.). Ninguno de los individuos estaba enfermo o presentaba abscesos en relación con sus atributos morfológicos. Según los resultados, el manejo empleado no representa un riesgo para esta población de reptiles.

Palabras clave: parasitología; microbiología; ambiente; salud; laguna.

\section{Abstract}

The objective of this study was to determinate blood parameters and the presence of parasites in a wild population of tortugas deslizantes (Trachemys scripta emolli) during the dry season of 2013 in the Caño Negro Wildlife Refuge in Costa Rica. Individuals were captured from different gaps using nets. Blood samples were taken to determine chemical parameters of blood and hematocrit, as well as morphological values and body conditions. In addition, samples of feces and ectoparasites were collected. The hematocrit $\mathrm{Cl} 95.0 \%$ was $[14.7091,18.7356]$ and no significant difference were found between sexes. The $95 \% \mathrm{Cl}$ for blood chemistry parameters were: AST (U/L) [87.6214, 148.929], CK (U/L) [1439.2, 2487.08], AU (umol/L) [68.22, 90.43 ], GLU (mmol/L) [4.26, 5.47], PHOS [0.46, 0.61], Ca (mmol/L) [1.99, 2.15], PT (g/L) [22.16, 24.44], $\mathrm{K}(\mathrm{mmol} / \mathrm{L})[3.31,3.83]$, and $\mathrm{Na}$ (mmol/L)[127.92, 130.93]. A total of 21 fecal samples was obtained, of which 10 were positive for parasites (23.8\% Strongyloides, 14.3\% Ascaridia, 9.5\% Coccidia, and 9.5\% Pentastomid). A leech from the Rhynchobdellidae family was the only ectoparasite found (Placobdella sp.). None of the individuals were ill or had abscesses in relation to their morphological attributes. Based on the results, the handling used does not represent a risk for this reptile population.

Keywords: parasitology; microbiology; environment; health status; lagoon.

\section{Resumo}

0 objetivo deste trabalho é determinar os parâmetros sanguíneos e a presença de parasitas em uma população selvagem de tortugas deslizantes (Trachemys scripta emolli) durante a estação seca de 2013 no Refúgio de Vida Selvagem Caño Negro, Costa Rica. Usando redes, os indivíduos foram capturados de diferentes tocas. Foram coletadas amostras de sangue para determinar os parâmetros químicos e 0 hematócrito, assim como dos valores morfológicos e das condições corporais. Além disso, foram coletadas amostras de fezes e ectoparasitas. O IC do hematócrito de 95\% foi [14 7091, 18 7356] e não foram encontradas diferenças significativas entre os sexos. $0 \mathrm{IC}$ de $95 \%$ nos parâmetros de química do sangue foram: AST(U/L)[87 6214, 148 929], CK (U/L)[1439.2, 2487.08], AU (umol/L)[68.22, 90.43 ], GLU (mmol/L) $[4.26,5.47]$, PHOS [0.46, 0.61], Ca (mmol/L) [1.99, 2.15], PT (g/L) [22.16, 24.44], K (mmol/L) [3.31, 3.83] e Na (mmol/L) [127.92, 130.93]. Havia também 21 amostras de fezes, das quais 10 eram positivas para parasitas (23.8\% de estrongiloidose, $14.3 \%$ de ascarídea, $9.5 \%$ de coccídea e $9.5 \%$ de pentastomida). Uma sanguessuga da família Rhynchobdellida foi o único ectoparasita encontrado (Placobdella sp.). Nenhum dos indivíduos estava doente ou teve abscessos em relação aos seus atributos morfológicos. De acordo com os resultados, o manejo empregado não representa um risco para essa população de répteis.

Palavras-chaves: parasitologia; microbiologia; ambiente; saúde; lagoa. 


\section{Introducción}

En Costa Rica, a pesar de ser ilegal la captura de especies silvestres (Reglamento a la ley de conservación de la vida silvestre, n. ${ }^{\circ} 7317$, artículo 7. Veda de Fauna Silvestre), muchas de ellas son capturadas en su entorno natural cuando son recién nacidas, para luego ser incorporadas a los hogares como mascotas y los reptiles son un ejemplo muy popular. En el 2001, la Fundación Mundial de Vida Silvestre (WWF, por sus siglas en inglés) estimó que más de 640000 reptiles son comercializados cada año. Sin embargo, según reportes posteriores, se consideró que esta cifra era realmente baja, de acuerdo con la realidad (Smith, Schloegel y Rosen, 2012).

Costa Rica posee aproximadamente 222 especies de reptiles, de las cuales ocho son tortugas continentales. Una de ellas es la Trachemys scripta, que comprende dos subespecies: T. scripta emolli, encontrada solo en una pequeña porción del norte del país y en Nicaragua, y $T$. scripta venusta, ampliamente distribuida a lo largo de América Central (Seidel, 2002). T. s. emolli es una especie esencialmente acuática y de hábitos diurnos, cuya dieta varía según su edad: de adultos son omnívoros, pero su dieta primordial es de plantas acuáticas; de juveniles se alimentan de invertebrados en gran cantidad. La época reproductiva es principalmente de setiembre a noviembre; sin embargo, las hembras ponen huevos durante todo el año. Los principales depredadores de sus huevos son gran variedad de mamíferos, aves, lagartijas e insectos, además del ser humano (Méndez, 2005; Savage, 2002).

T. s. emolli es la especie de tortuga más distribuida en los hogares costarricenses debido, en parte, a permisos de colecta que el Estado brinda a pobladores de los alrededores del Refugio de Vida Silvestre Caño Negro.
En general, dichos consentimientos autorizan vender un $70 \%$ del total de los nacimientos obtenidos de los huevos colectados en el refugio, mientras el restante $30 \%$ se libera en el mismo humedal. La asociación encargada cuenta con un vivero donde se trasladan las nidadas recolectadas del medio (Méndez, 2005). Tomando en cuenta las características de este manejo, se requiere datos que faciliten la determinación de potenciales riesgos sanitarios para la salud pública y las poblaciones silvestres, sobre todo, considerando que el sistema inmunitario de las tortugas podría depender de la temperatura del ambiente, del estrés, de la humedad ambiental, la deforestación o la alimentación (Abarca, López del P., Peña y López, 2011; Martínez-Silvestre y Soler-Massana, 2008).

La infección con parásitos juega un rol importante en la aparición de enfermedades, debido a que el estrés, la alta concentración de individuos, el confinamiento, el compartir el espacio con otras especies, entre otros, pueden provocar un aumento en la carga y diversidad parasitaria, así como una disminución en la respuesta del sistema inmune de las especies; por ende, la propagación de enfermedades. Esta situación se puede presentar tanto en animales en cautiverio como en aquellos de vida libre, en donde la sequía, deforestación, depredación y los cambios antropogénicos que incrementan el contacto entre humanos y vida silvestre, así como otros factores, pueden generar condiciones adversas para los individuos (Mafuyai, Barshep, Audu, Kumbak y Ojobe, 2013; Rataj, Lindtner-Knific, Vlahović, Mavri y Dovč, 2011; Rideout, Montali, Phillips y Gardiner, 1987). En ese sentido, la interpretación hematológica en reptiles es una excelente herramienta para el diagnóstico de patologías en estas especies (Martínez-Silvestre, Lavín y Cuenca, 2011). 
A pesar de su utilidad en los programas de manejo, el país cuenta con limitados valores referenciales para animales silvestres. Por ejemplo, aunque se cuenta con algunos para T. scripta elegans (FRCVS y DVM, 2005; Hidalgo-Vila, Martínez-Silvestre y Díaz-Paniagua, 2006), existe carencia de datos sobre los parásitos intestinales, hematocrito y química sanguínea para T. s. emolli.

\section{Metodología}

Área de estudio: La toma de muestras se realizó en el humedal del Refugio Nacional de Vida Silvestre Caño Negro (RNVSCN), Costa Rica, en el verano del 2013 (coordenadas geográficas $10^{\circ} 48^{\prime} 12^{\prime \prime}$ y $10^{\circ} 57^{\prime} 21^{\prime \prime}$ latitud norte, $-84^{\circ} 42^{\prime} 30^{\prime \prime}$ y $-84^{\circ} 45^{\prime} 50^{\prime}$ ' longitud oeste). Este sitio se encuentra entre los $30 \mathrm{y}$ $44 \mathrm{msnm}$, posee una extensión de 9969 ha, de los cuales aproximadamente 3500 ha corresponden a humedales. Cerca de $10 \%$ de su territorio está cubierto por una laguna estacional que, durante la breve estación seca, llega a desaparecer casi por completo, formando una serie de lagunas estacionales variables en su profundidad y extensión, según los cambios en el caudal del río Frío y sus tributarios, escenario que también afecta la disponibilidad de hábitat para los organismos que allí se encuentran (Coloma, Caballero, Ricart y Bueno, 2009; Pacheco, Rodríguez, López y Canessa, 1985; Rojas, 2014; Ruiz, 1995). El área se caracteriza por tener bosques tropicales muy húmedos y, dado su papel regulador de inundaciones y sostén de caudales de los principales ríos o caños, influye en el mantenimiento de la calidad ambiental de la zona Caribe Norte (Coloma et al., 2009).

La colecta de las tortugas se realizó en los ríos Frío y Caño Blanco, así como en las lagunas San Sebastián I, San Sebastián II,
Caño Romero, Romero, Deshuesadero, El Guabal, El Guabo, Charco Verde, San Jerónimo y Apante (ver figura 1).

Captura de individuos. El tamaño de la muestra fue de 80 individuos de una población de la subespecie $T$. s. emolli de vida libre del RNVSCN, capturados entre los meses de marzo y abril del 2013 (época seca), bajo el Consentimiento Previamente Informado (CPI) con la Comisión Nacional para la Gestión de la Biodiversidad (CONAGEBIO). La colecta se dio en nueve de las 15 lagunas encontradas en ese periodo, en dos ríos y un caño. Las lagunas tenían una profundidad de entre $30 \mathrm{~cm}$ y $1 \mathrm{~m}$ y contaban con tamaños similares (diámetro aproximado de $50 \mathrm{~m}$ ), con aguas tranquilas y presencia tanto de algas como de lirios acuáticos.

La técnica de captura utilizada consistía en sumergir en el agua redes de un diámetro de unos $50 \mathrm{~cm}$, cuando se observaba una tortuga sacando la cabeza para respirar y cuando el agua era transparente, junto con un trasmallo. Esta técnica se utilizó en sitios con profundidades que oscilaron entre 1 y 2 $\mathrm{m}$. Cuando el nivel del agua fue muy bajo, se capturaron manualmente. En los sectores de río, se recurrió a la caña de pescar con anzuelo. Las capturas se llevaron a cabo en periodos de tres días, durante tres fines de semana. Posteriormente, las tortugas fueron liberadas en el río.

Cada tortuga fue identificada mediante la inscripción sobre el caparazón, con esmalte de uñas rojo, de un número del 1 al 80, para evitar la recaptura. Además, los integrantes de la asociación buscaron muescas hechas previamente por ellos, las cuales se realizaban durante las liberaciones de los individuos nacidos en cautiverio, cuando su caparazón medía 7 cm de largo. 


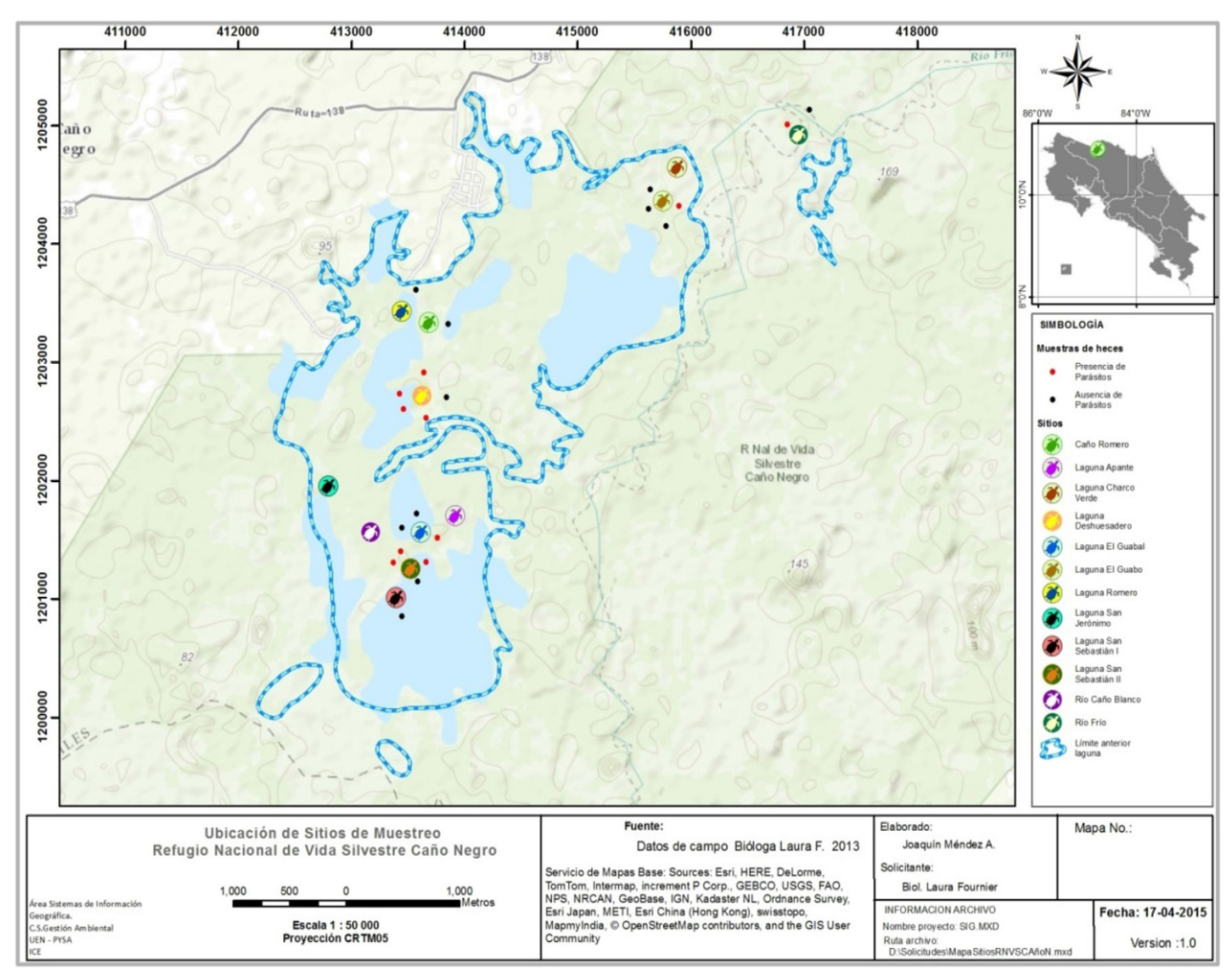

Figura 1. Sitios de colecta de tortugas Trachemys scripta emolli en el Refugio de Vida Silvestre Caño Negro, entre marzo y abril del 2013, y resultados de hallazgos de coprológicos, donde los puntos rojos significan "individuo con parásitos" y los negros “individuo sin parásitos”. Nota: Ver autoría en la imagen.

Morfometría. Utilizando una cinta métrica flexible, se tomaron los siguientes datos morfométricos: largo curvo del caparazón (LCC), ancho curvo del caparazón (ACC), largo curvo del plastrón (LCP) y ancho curvo del plastrón (ACP). Con estas medidas, se determinó su edad, de acuerdo con un modelo propuesto previamente por Savage (2002), en el cual los machos adultos miden entre 120 y $340 \mathrm{~mm}$ y las hembras adultas entre 158 y $600 \mathrm{~mm}$. Según esto, los animales fueron clasificados como adultos o juveniles.
$\mathrm{Su}$ peso (g) se determinó con una balanza electrónica marca UWE, modelo AXM-30K, mientras que el sexo fue identificado con base en caracteres secundarios, como el tamaño de la cola, la distancia precloacal y la concavidad plastral.

Colecta de sangre. La obtención de sangre para la evaluación del análisis clínico se hizo a través de la inserción de una jeringa de $5 \mathrm{ml}$ con una aguja de $22 \mathrm{G} \times 1$ $1 / 2$ " en el seno del subcaparazón. El procedimiento solo tomó unos pocos minutos y las tortugas no mostraron ninguna evidencia 
de malestar después del procedimiento. Las variables de estudio fueron: hematocrito, aspartatoaminotransferasa (AST), ácidos biliares (BA), creatina quinasa $(\mathrm{CK})$, ácido úrico (AU), glucosa (GLU), fósforo (PHOS), calcio $(\mathrm{CA}++)$, proteína total $(\mathrm{TP})$, albúmina (ALB), globulina (GLOB), potasio $(\mathrm{K}+)$ y sodio $(\mathrm{NA}+)$.

La cantidad de sangre se distribuyó de la siguiente manera: $1 \mathrm{ml}$ en tubos con heparina para hematocrito y $3 \mathrm{ml}$ en tubos sin ningún reactivo. Se obtuvo al menos $100 \mu 1$ de suero por muestra, el cual se almacenó en tubos estériles debidamente rotulados con el número de tortuga, la fecha de toma de la muestra y el lugar de muestreo. Las muestras de sangre con heparina se colocaron verticalmente y se dejaron reposar 20-30 min a temperatura ambiente. Luego, se colocaron en la misma posición a $4{ }^{\circ} \mathrm{C}$ hasta su análisis, efectuado como máximo tres días después de su toma en la Escuela de Medicina Veterinaria de la Universidad Nacional. El equipo utilizado para la medición del hematocrito fue Sorvail Legend Micro 17 Centrifuge, de Thermo Electronic Corporation. La medición de la química sanguínea se realizó para 48 muestras, mediante reactores de perfil de reptil VetScan, marca ABAXIS, serie \# 0000V11778, que utiliza reactivos secos y líquidos para proporcionar determinaciones cuantitativas in vitro de las variables.

Los resultados obtenidos fueron analizados mediante estadística descriptiva y, con la prueba de Kruskall Wallis, se comprobó si existía diferencia en la cantidad de hematrocito entre machos y hembras adultos, así como en los datos de química sanguínea. Las pruebas se realizaron utilizando el paquete estadístico Statgraphis Centurion.

A todos los animales capturados se les midió el hematocrito y a 48 de estos se les analizó su química sanguínea. Con el propósito de obtener el número de muestras necesario para que el análisis fuera representativo, se tomaron datos de hematocrito para Trachemys scripta elegans y se le calculó el coeficiente de variación. El tamaño de muestra requerido para establecer intervalos de referencia, según la Asociación Norteamericana de Patólogos Clínicos Veterinarios (ASVCP), es 40 (Friedrichs et al., 2012). La comparación entre los individuos nacidos en cautiverio con los datos generales obtenidos de química sanguínea se realizó con los machos, ya que de los diez individuos nacidos en cautiverio nueve fueron machos y solo una hembra. Sin embargo, cuando el resultado de alguno de los parámetros medidos en la hembra no se ajustó a los datos obtenidos por los machos, se relacionó con la información de las hembras.

Colecta de muestras de heces. Los animales se colocaron en recipientes individuales, por un máximo de un día, hasta que depositaran sus heces. Las muestras obtenidas se pusieron en viales de 1,5 ml. Cada muestra se rotuló con el número de tortuga y se mantuvo a $4{ }^{\circ} \mathrm{C}$ hasta ser analizada en el Laboratorio de Parasitología de la Escuela de Medicina Veterinaria de la Universidad Nacional, en un plazo no mayor a ocho días. Para esto, se utilizó el método de Sheather modificado, el cual es un método cualitativo (Hernández, 2010).

Colecta de ectoparásitos. Cada tortuga fue revisada minuciosamente en busca de ectoparásitos, los cuales fueron extraídos mediante pinzas y preservados en viales con alcohol al $75 \%$. Cada vial fue etiquetado con fecha, número de individuo y lugar de colecta. 


\section{Resultados}

Se tomaron 80 muestras de sangre, de las cuales resultaron apropiadas 75. Diez de ellas correspondían a individuos nacidos en cautiverio y liberados a los pocos meses de nacidos. El hematocrito obtenido para hembras y machos, con un intervalo de confianza al $95 \%$, fue de $[14.5923 ; 20.5975]$ y [13.3699; 16.9723], respectivamente. No se encontró diferencia significativa entre sexos $(\mathrm{p}=0.1657)$.

De las 48 muestras obtenidas para química sanguínea, únicamente fue posible analizar 40, debido a que las restantes se contaminaron con linfa. Las muestras provenían de 22 machos y 18 hembras, de las cuales siete pertenecían a individuos nacidos en cautiverio. Únicamente para el fósforo $(p=0.05)$ hubo diferencias significativas entre machos y hembras (ver tabla 1).

Al comparar los datos obtenidos de los individuos nacidos en cautiverio y liberados con los datos generales, se encontró que todos los valores del $\mathrm{Ca}, \mathrm{K}$ y $\mathrm{Na}$ fueron similares. Sin embargo, cinco machos y la única hembra muestreada de ese grupo presentaban los siguientes datos extremos: en el caso de la hembra, AST $=341 \mathrm{U} / \mathrm{L}$, $\mathrm{AU}=120 \mu \mathrm{mol} / \mathrm{L}, \mathrm{PHOS}=0.84 \mathrm{mmol} / \mathrm{L} \mathrm{y}$ $\mathrm{PT}=4.39 \mathrm{~g} / \mathrm{L}$; uno de los machos mostraba $\mathrm{CK}=820 \mathrm{U} / \mathrm{L}, \mathrm{AU}=47 \mu \mathrm{mol} / \mathrm{L}$ y GLU $=7.1 \mathrm{mmol} / \mathrm{L}$, en tanto que otro tenía $\mathrm{CK}$ $=767 \mathrm{U} / \mathrm{L}$ y $\mathrm{AST}=43 \mathrm{U} / \mathrm{L}$; los otros tres machos obtuvieron solo un valor extremo $($ Hto $=8.39 \%, 37 \mathrm{AU}=125 \mu \mathrm{mol} / \mathrm{L} \mathrm{y} \mathrm{CK}$ $=841 \mathrm{U} / \mathrm{L}$, respectivamente).

De las 21 deposiciones analizadas, 12 fueron de hembras y nueve de machos. Provenían de río Frío (2), laguna San Sebastián I (1), laguna San Sebastián II (4), Caño Romero (1), laguna Romero (1), laguna Deshuesadero (5), El Guabal (3) y El Guabo (4). No se obtuvo muestras de las lagunas Charco Verde, San Jerónimo y Apante ni del río Caño Blanco (figura 1). En total, se encontraron 12 parásitos, que venían de 10 reptiles, agrupados en cuatro grupos distintos: Strongyloidea (5, 23.8 $\%)$, Ascaridia (3, $14.3 \%)$, Coccidia $(2,9.5$ $\%$ ) y Pentastomido $(2,9.5 \%)$. Nueve individuos presentaron solo una especie de parásito y solo uno tenía tres distintos. Únicamente se colectó una muestra de heces de un individuo criado en cautiverio por la asociación ULIMA (palabra maleku que significa tortuga), el cual no presentó ningún parásito. Exclusivamente una hembra adulta colectada en la laguna San Sebastián II presentó una sanguijuela en su ano, la cual era de la especie Placobdella sp., familia Rhynchobdellidae.

Con respecto a la cantidad de muestras positivas por sitio de muestreo, la laguna San Sebastián II y la laguna Deshuesadero obtuvieron el mayor porcentaje de aquellas, con tres y cuatro muestras de parásitos positivas, respectivamente; sin embargo, fueron en las que más excretas se recolectaron. Las lagunas Guabal y el Guabo, así como el río Frío, obtuvieron una muestra positiva cada uno. Las tomas de los quelonios de las lagunas San Sebastián I, Romero y Caño Romero no presentaron parásitos, aunque cabe destacar que solo se analizó una muestra de heces para cada uno de estos tres sitios de muestreo. 


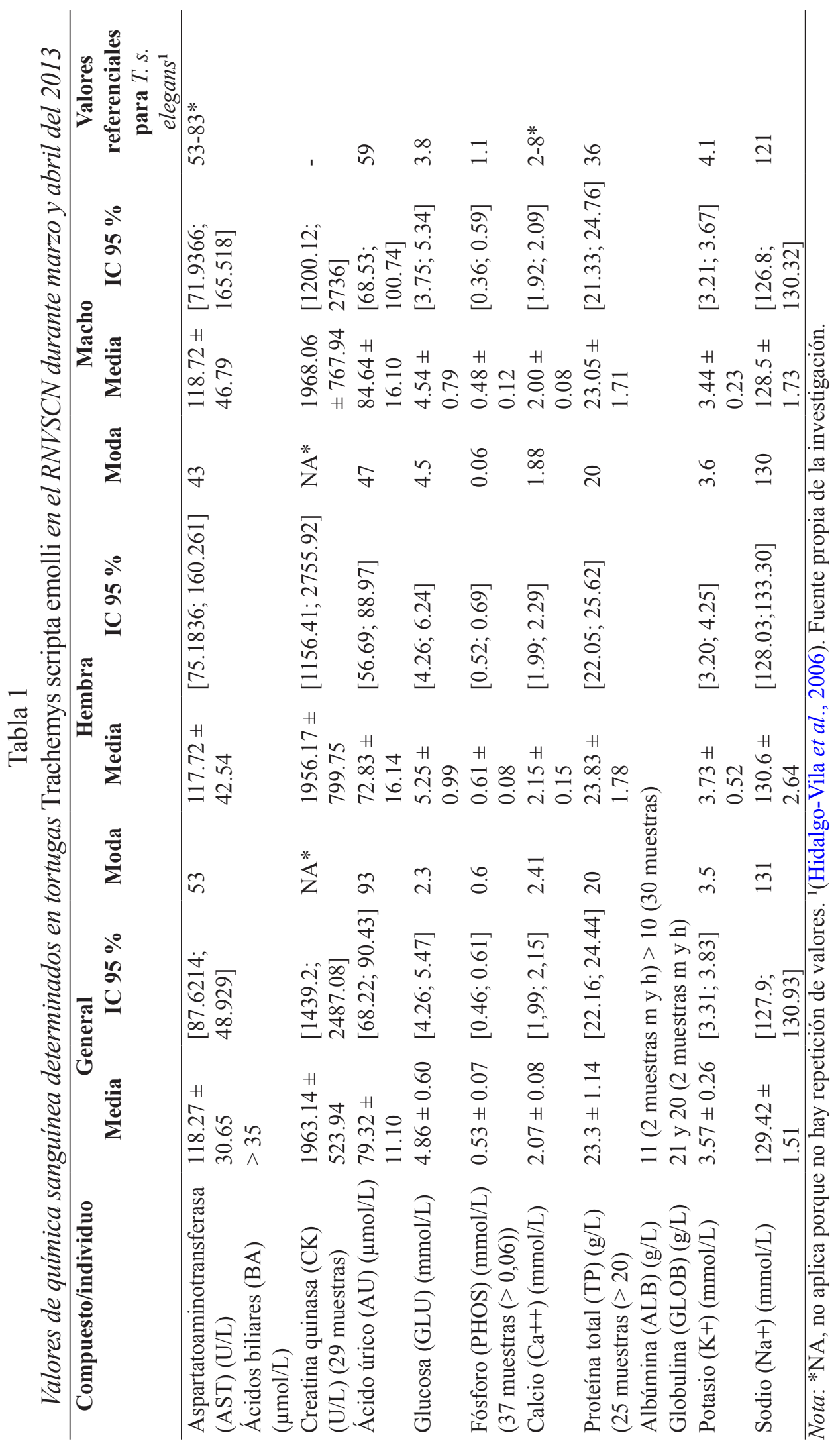




\section{Discusión}

Para la conservación efectiva de una especie, se requiere la incorporación activa de la población humana en los proyectos relacionados. Un ejemplo lo constituye la tortuga de río T. s. emolli del Refugio de Vida Silvestre Caño Negro, en el que un grupo de la población (la asociación ULIMA), al verse beneficiado con su conservación, intenta protegerla en lugar de cazarla para consumo, como ha pasado con la Podocnemis sextuberculata en Sur América (Miorando, Rebêlo, Pignati y Pezzuti, 2013). Además, el área de estudio está protegida y hay periodos de veda para la pesca (principalmente de la especie Atractosteus tropicus - pez gaspar-), lo que favorece la supervivencia de las tortugas, pues, durante dicha actividad, pueden ser atraídas por el anzuelo, según lo reportaron integrantes de la asociación. También, está prohibido pescar con trasmallo, técnica con la cual igual se ven afectados estos reptiles al quedar atrapados.

Tomando en consideración el manejo implementado en la zona, es necesario generar información que permita identificar no solamente si las acciones desarrolladas producen algún impacto, sino riesgos eventuales para las personas de la asociación ULIMA que lo ejecutan.

Aunque la interpretación de valores hematológicos se ha utilizado en quelonios para diagnosticar diferentes estados patológicos y evaluar su estado de salud (Hidalgo-Vila et al., 2006), su interpretación precisa se dificulta, debido a la gran variabilidad en los resultados, asociada a factores como edad, sexo, estado reproductivo, dieta, tamaño, condiciones ambientales, posición geográfica y metodología usada (Prieto-Torres et al., 2012). En este sentido, en el presente estudio no se halló diferencias para la química sanguínea ni para el hematocrito entre machos y hembras, excepto para el PHOS. Esto concuerda con hallazgos previos en T. s. callirostris en Colombia (Navarro, Real, Rincón, Villamizar y Arcila, 2005) y en una especie de tortuga terrestre argentina (Chelonoidis chilensis chilensis) (Troiano y Silva, 1998).

De acuerdo con los resultados obtenidos para parásitos, se podría inferir que la población de T. s. emolli del Refugio de Vida Silvestre Caño Negro es sana, sobre todo si se compara con los datos reportados para animales en cautiverio (Rataj et al., 2011; Rideout et al., 1987; Ruiz, 2000). Esto podría ser una señal positiva sobre la crianza de la especie, pero los resultados alcanzados no son concluyentes, dado el bajo número de heces obtenidas.

Resulta importante destacar que los datos de las tortugas liberadas por la asociación ULIMA fueron semejantes a los de las nacidas en cautiverio, en cuanto a hematocrito y química sanguínea, por lo que se podría concluir que el manejo, la colecta de huevos y la posterior liberación de las tortugas no han generado impacto en los individuos, de acuerdo con los parámetros obtenidos en este estudio. De igual forma, el encontrar individuos adultos previamente liberados por la asociación en su etapa juvenil es otro indicador de un buen manejo por parte de esta asociación. Cabe destacar, además, que muy pocos individuos se observaron con amputaciones o daños, los cuales son propios de alguna lucha por escape, y ninguno se observó enfermo ni con abscesos. Tampoco se encontró animales con signos de enfermedad, lo cual podría estar relacionado con la no presencia de plaguicidas (Fournier et al., 2018) o con la regulación de la calidad del agua por parte de los humedales (Nahlik y Mitsch, 2006). 
Dados los resultados conseguidos, se podría concluir que el manejo realizado por la asociación al momento de la investigación no representa un riesgo mayor para dicha población. Esto concuerda con los datos generados en otra indagación, en la cual se capturó 523 tortugas en 13 sitios distintos (Rojas, 2014).

\section{Agradecimientos o reconocimientos}

Al ingeniero agrónomo Raúl Fournier Zepeda y a la asociación ULIMA por su apoyo durante la captura de las tortugas.

\section{Referencias}

Abarca, K.; López del P., J.; Peña, A. y López, J. C. (2011). Tenencia y estado de salud de mascotas de niños inmunocomprometidos, con énfasis en enfermedades zoonóticas. Revista chilena de infectología, 28(3), 205-210. https:// doi.org/10.4067/S0716-10182011000300001

Coloma, C.; Caballero, L.; Ricart, M. y Bueno, S. (2009). Diagnóstico del Refugio de Vida Silvestre Caño Negro Arenal-Huetar Norte Costa Rica. En Memoria del proyecto final de carrera de Ciencias Ambientales (pp. 1- 194). Costa Rica: UAB. Recuperado de https:// core.ac.uk/download/pdf/13301674.pdf

Fournier, M. L.; Echeverría-Sáenz, S.; Mena, F.; Arias-Andrés, M.; De la Cruz, E. y Ruepert, C. (2018). Risk assessment of agriculture impact on the Frío River watershed and Caño Negro Ramsar wetland, Costa Rica. Environmental Science and Pollution Research, 25(14), 13347-13359. https://doi. org/10.1007/s11356-016-8353-y

FRCVS, S. J. D. Bv. Dz. D. D. y DVM, D. R. M. M. (2005). Reptile Medicine and Surgery (2.a edición). St. Louis, Mo: Saunders.

Friedrichs, K. R.; Harr, K. E.; Freeman, K. P.; Szladovits, B.; Walton, R. M.; Barnhart, K. F. y Blaco, J. (2012). ASVCP reference interval guidelines: determination of de novo reference intervals in veterinary species and other related topics. Veterinary Clinical
Pathology, 41(4), 441-453. https://doi. org/10.1111/vcp.12006

Hernández, J. (2010). Técnicas parasitológicas. Heredia, Costa Rica: Cátedra inédita de Parasitología y Enfermedades Parasitarias, Escuela de Medicina Veterinaria.

Hidalgo-Vila, J.; Martínez-Silvestre, A. y Díaz-Paniagua, C. (2006). Bening ovarian teratoma in a red-eared slider turtle (Trachemys scripta elegans). The Veterinary Record, 159(4), 122123. http://dx.doi.org/10.1136/vr.159.4.122

Mafuyai, H. B.; Barshep, Y.; Audu, B. S.; Kumbak, D. y Ojobe, T. O. (2013). Baboons as potential reservoirs of zoonotic gastrointestinal parasite infections at Yankari National Park, Nigeria. African Health Sciences, 13(2), 252-254. Recuperado de https://www.researchgate. net/publication/258530652_Baboons_as_potential_reservoirs_of_zoonotic_gastrointestinal_parasite_infections_at_Yankari_National_Park_Nigeria

Martínez-Silvestre, A. y Soler Massana, J. (2008). Enfermedades infecciosas y parasitarias en tortugas. Consulta Difusión Veterinaria, 150, 43-54. Recuperado de https://www.researchgate.net/publication/260435197_Enfermedades_infecciosas_y_parasitarias_en_tortugas

Martínez-Silvestre, A.; Lavín, S. y Cuenca, R. (2011). Hematología y citología sanguínea en reptiles. Clínica Veterinaria de Pequeños Animales, 31(3), 131-141. Recuperado de https://ddd.uab.cat/pub/clivetpeqani/clivetpeqani_a2011v31n3/clivetpeqaniv31n3p131.pdf

Méndez, C. (2005). Plan de Manejo Zoocriadero Trachemys scripta RNVSCN. Caño Negro, Costa Rica: Asociación ULIMA.

Miorando, P. S.; Rebêlo, G. H.; Pignati, M. T. y Brito Pezzuti, J. C. (2013). Effects of Community-Based Management on Amazon River Turtles: A Case Study of Podocnemis sextuberculata in the Lower Amazon Floodplain, Pará, Brazil. Chelonian Conservation and Biology, 12(1), 143-150. https://doi. org/10.2744/CCB-1011.1

Nahlik, A. M. y Mitsch, W. J. (2006). Tropical treatment wetlands dominated by free-floating macrophytes for water quality improvement in Costa Rica. Ecological Engineering, 28(3), 246-257. https://doi.org/10.1016/j. ecoleng.2006.07.006 
Navarro, R.; Real, W.; Rincón, M.; Villamizar, M. y Arcila, V. (2005). Especies Silvestres. Revista Colombiana de Ciencias Pecuarias, 18(4), 373-374. Recuperado de http://www.redalyc. org/pdf/2950/295022975014.pdf

Pacheco, A.; Rodríguez, M.; López, E. y Canessa, G. (1985). Plan operativo. Refugio Nacional de fauna Silvestre Caño Negro. San José, Costa Rica: Subdirección General de Vida Silvestre, Ministerio de Agricultura y Ganadería.

Prieto-Torres, D. A.; Hernández-Rangel, J. L.; Bravo-Henrique, A. R.; Alvarado-Árraga, M. C.; Dávila-Ojeda, M. J. y Quiroz-Sánchez, N. R. (2012). Valores hematológicos de la población anidadora de tortuga verde (Chelonia mydas) en el refugio de fauna silvestre Isla de Aves, Venezuela. Revista Científica, 12 (3), 273-280. Recuperado de http://www.redalyc. org/articulo.oa?id=95922219011

Rataj, A. V.; Lindtner-Knific, R.; Vlahović, K.; Mavri, U. y Dovč, A. (2011). Parasites in pet reptiles. Acta Veterinaria Scandinavica, 53(33), 1-20. Recuperado de https:// actavetscand.biomedcentral.com/track/ pdf/10.1186/1751-0147-53-33

Rideout, B. A.; Montali, R. J.; Phillips, L. G. y Gardiner, C. H. (1987). Mortality of captive tortoises due to viviparous nematodes of the genus Proatractis (Family Atractidae). Journal of Wildlife Diseases, 23(1), 103108. Recuperado de https://repository.si.edu/ bitstream/handle/10088/4294/Montali1987. pdf? sequence $=1$ \&isAllowed $=\mathrm{y}$

Rojas, E. (2014). Estructura poblacional, supervivencia y abundancia de la Tortuga Verde de Río Trachemys emolli (Testudines: Emydidae) manejada en el Refugio Nacional de Vida Silvestre Caño Negro. San José, Costa Rica: UNED.

Ruiz, A. (1995). Ecología de Anidación de la Tortuga Trachemys scripta (Testudines: Emydidae) en la Laguna San Sebastián, Caño Negro, Costa Rica. Recuperado de http://www.sinac.go.cr/
ES/partciudygober/Sistematizacion $\% 20$ Experiencias/INFORME\%209\%20-\%20 ULIMA $\% 20 \mathrm{CA} \% \mathrm{C} 3 \% 910 \% 20$ NEGRO.pdf

Ruiz, P. A. (2000). Prevalencia Parasitaria en Tortugas Geochelone carbonaria y Geochelone denticulate en el Centro de Recepción y Rehabilitación de fauna Silvestre del Dama en Engativá. Recuperado de http://www.ambientebogota.gov.co/c/document_library/get_file?uuid=8eb86719-8fa4-4f33-9324-377a5ecd755c\&groupId $=10157$

Savage, J. M. (2002). The Amphibians and Reptiles of Costa Rica: a Herpetofauna between Two Continents, Between Two Seas. University of Chicago Press. Recuperado de https://www. researchgate.net/publication/37695427_The Amphibians_and_Reptiles_of_Costa_Rica_A_Herpetofauna_Between_Two_Continents_Between_Two_Seas

Seidel, M. E. (2002). Taxonomic Observations on Extant Species and Subspecies of Slider Turtles, Genus Trachemys. Journal of Herpetology, 36(2), 285-292. https://doi. org/10.2307/1566003

Smith, K. F.; Schloegel, L. M. y Rosen, G. E. (2012). Wildlife Trade and the Spread of Disease. En A. A. Aguirre; R. S. Ostfeld y P. Daszak (eds.), New Directions in Conservation Medicine: Applied Cases of Ecological Health (pp. 151163). Oxford, New York: Oxford University Press. Recuperado de https://global.oup.com/ academic/product/new-directions-in-conservation-medicine-9780199731473?c$\mathrm{c}=$ us\&lang $=\mathrm{en} \&$

Troiano, J. C. y Silva, M. C. (1998). Valores hematológicos de referencia en tortuga terrestre -argentina (Chelonoidis chilensis chilensis). Analecta Veterinaria, 18, (1-2), 47-51. Recuperado de http://sedici.unlp.edu.ar/bitstream/ handle/10915/11095/Documento_completo. pdf? sequence $=1$ \&isAllowed $=y$

\section{(9) $(1 \otimes \Theta$}

Determinación de intervalos básicos de salud en tortugas resbaladoras (Trachemys scripta emolli) del Refugio de Vida Silvestre Caño Negro, Costa Rica (Laura Fournier-Gutiérrez y otros) in Uniciencia is protected by Attribution-NonCommercial-NoDerivs 3.0 Unported (CC BY-NC-ND 3.0) 